\title{
Finite Element Modelling of SAW Correlator
}

\author{
Ajay C.Tikka ${ }^{a}$ Said F.Al-Sarawi ${ }^{a}$ and Derek Abbott $^{b}$ \\ ${ }^{a}$ Centre for High Performance Integrated Technologies and Systems (CHiPTec), The University \\ of Adelaide, SA 5005, Australia; \\ ${ }^{b}$ Centre for Biomedical Engineering, The University of Adelaide, SA 5005, Australia
}

\begin{abstract}
Numerical simulations of SAW correlators so far are limited to delta function and equivalent circuit models. These models are not accurate as they do not replicate the actual behaviour of the device. Manufacturing a correlator to specifically realise a different configuration is both expensive and time consuming. With the continuous improvement in computing capacity, switching to finite element modelling would be more appropriate. In this paper a novel way of modelling a SAW correlator using finite element analysis is presented. This modelling approach allows the consideration of different code implementation and device structures. This is demonstrated through simulation results for a $5 \times 2$-bit Barker sequence encoded SAW correlator. These results show the effect of both bulk and leaky modes on the device performance at various operating frequencies. Moreover, the ways in which the gain of the correlator can be optimised though variation of design parameters will also be outlined.
\end{abstract}

Keywords: Surface Acoustic Wave (SAW) Correlator, Finite Element Analysis (FEA), Bulk Acoustic Wave (BAW), Leaky Surface Acoustic Wave (LSAW), Barker Sequence.

\section{INTRODUCTION}

Complex microwave and digital signal processing functions can be combined into a single component using a SAW correlator. ${ }^{1}$ They can be used as wirelessly interrogatable and completely passive devices in many hostile environments, as power sensors and actuators, in a secure manner. Precise characterization of acoustic mode propagation determined by the encoded code sequence, device geometry and material characteristics is crucial for designing a SAW correlator. This is commonly performed by manufacturing a test structure, and then examining the behaviour of the device through measurements. Such an approach is both time consuming and expensive as each device optimisation would require fabricating a test structure. On the other hand, numerical methods, mostly based on the delta function and equivalent circuit models have been developed to optimize the design process. ${ }^{2} \quad$ These models introduce simplifications or assumptions that are often invalid for actual designs. Even though this results in reduced computational time, this approach does not accurately model the correlator response. Furthermore, the second order effects such as backscattering, diffraction, charge distribution and mechanical loading are either simplified or neglected. ${ }^{3}$

Field theory is the most appropriate approach for designing SAW devices as it involves the resolution of all the partial differential equations for a given excitation. ${ }^{4}$ The finite element method (FEM) allows for accurate representation of field theory where the piezoelectric behaviour of the SAW devices is discretised and numerically solved. The FEM modelling of SAW devices was previously limited to either periodic structures or simple structures with few IDT electrodes..$^{3-5}$ With the availability of software packages with large node handling capability and an increase in computing performance and memory capacity, switching to a direct FEM for numerical simulation of a SAW correlator appears to be efficient.

Bulk and Leaky SAW mode characterisation using FEM has been well addressed, in the past, using periodic structures. ${ }^{6,7}$ However, quantitative understanding of the effect these modes have on the processing gain and the electromechanical coupling of an acoustic device remains to be a challenging problem in FEM. This is mainly due to huge constraint imposed by such modelling techniques on the available computational resources. However,

Further author information: (Send correspondence to Ajay Tikka)

Ajay Tikka: E-mail: ajay.tikka@adelaide.edu.au

Said Al-Sarawi: E-mail: alsarawi@eleceng.adelaide.edu.au

BioMEMS and Nanotechnology III, edited by Dan V. Nicolau, Derek Abbott, Kourosh Kalantar-Zadeh, Tiziana Di Matteo, Sergey M. Bezrukov, Proc. of SPIE Vol. 6799, 679915, (2007) · 0277-786X/07/\$18 · doi: 10.1117/12.764795 
the recent improvements made on that front have made it possible to model a complete SAW correlator by neglecting features that contribute diminutively to the final output. In this research we present an analysis of the effect various acoustic modes have on performance of a correlator using a direct finite element model previously developed for the analysis of surface acoustic waves and extraction of Coupling-Of-Mode (COM) parameters. $^{8}$

In this paper, modelling of a 2-dimensional, $5 \times 2$-bit Barker sequence encoded SAW correlator is presented using FEM. In Section 2, we discuss briefly the SAW correlator operation, the various modelling techniques that were employed previously and the advantages of using FEM modelling. Then in Section 3, we present the SAW correlator design, where the dimensions of the structure, materials used and boundary conditions are discussed. After the description of the model, we present and discuss the simulation results in Section 4, using harmonic analysis. The admittance, output voltage response graphs and contour plots of displacement in the frequency domain are presented to illustrate the effect of bulk and leaky-SAW modes on the correlator performance for two different piezoelectric substrate thicknesses. Moreover, the correlator's response when there is a code mismatch is also discusses. Conclusion and future work are presented in Section 5.

\section{CORRELATOR OPERATION AND MODELLING TECHNIQUES}

SAW based devices are increasingly being used in digital communications for code discrimination. These devices, apart from being inherently rugged and reliable, can provide bi-phase coding and processing gain by the variation of finger geometry. A SAW correlator responds with a correlated peak to an interrogating signal with the correct frequency and correct code. This aspect of the correlator can be used to power sensors and actuators in a secure manner. The concept of using a SAW correlator for improving the security and actuation of the wireless controlled microvalve was discussed previously. ${ }^{9}$ The feasibility of such an approach is heavily influenced by the insertion losses in the correlator. The ways in which the device performance can be optimised for high gain operation will be explained in the subsequent sections.

\subsection{Correlator Operation}

A brief description of the correlator operation is provided here for the sake of clarity. A more detailed description of the same was presented in Ref. 9. A SAW correlator, as shown in Fig. 1, is a passive pulse compression filter that operates through the correlation of phase shifts in the transmitted RF signal. It consists of an input IDT and a coded output IDT deposited on top of a piezoelectric substrate. The input IDT transduces the coded input RF signal into an acoustic wave. The electrodes of the output IDT are phase coded during construction in such a way that the correlator converts the correctly phase coded acoustic wave to a RF modulated electrical pulse. The device will respond with a correlation peak only when the code of the interrogating RF pulse matches with the embedded code in the output IDT, thus enhancing the processing gain of the correlator by combining the code reception scheme with the high Q operation of a bandpass filter. ${ }^{10}$ For all other transmitted signals with different codes, even the one's excited at the same frequency, the correlator would respond with a pseudo-random noise. The coding scheme used in this work is Binary Phase Shift Keying (BPSK), where the incoming radio wave has $180^{\circ}$ degree phase transitions in its waveform. BPSK is the simplest form of phase coding that offers optimum signal to noise ratio. By converting the long input BPSK signal with a matched pattern into a short $\mathrm{RF}$ modulated pulse, the correlator can provide considerable process gain.

A coded SAW based communication system, as shown in the Fig. 2, consists of an expander IDT in the transmitter and a compressor IDT in the receiver. A narrow pulse or a sinusoidal waveform is fed to the expander IDT to generate a coded acoustic signal depending on the geometry of the expander IDT. These acoustic waves propagate through the substrate to the transmitting IDT, which transforms these coded acoustic waves to electrical coded RF signal. The output from the transmitting IDT is fed to an amplifier, to strengthen the signal, and then to a transmitting antenna. The receiver consists of a correlator, operating as discussed above, with its input IDT connected to a receiving antenna to intercept the transmitted coded RF signal. The expander in the transmitter is an exact replica of the compressor/coded IDT of the correlator. The coding of the expander and compressor determines the autocorrelation function performed by the correlator. The preferred coding scheme is Barker sequence, for reasons explained in our previous publication. ${ }^{9}$

Proc. of SPIE Vol. 6799 679915-2 


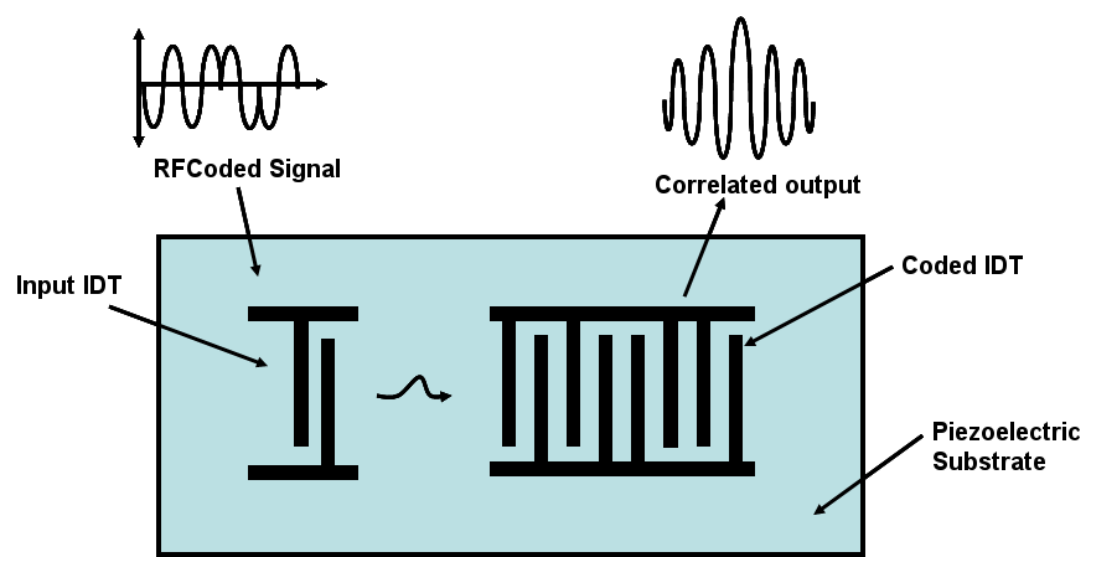

Figure 1. Surface acoustic wave correlator.
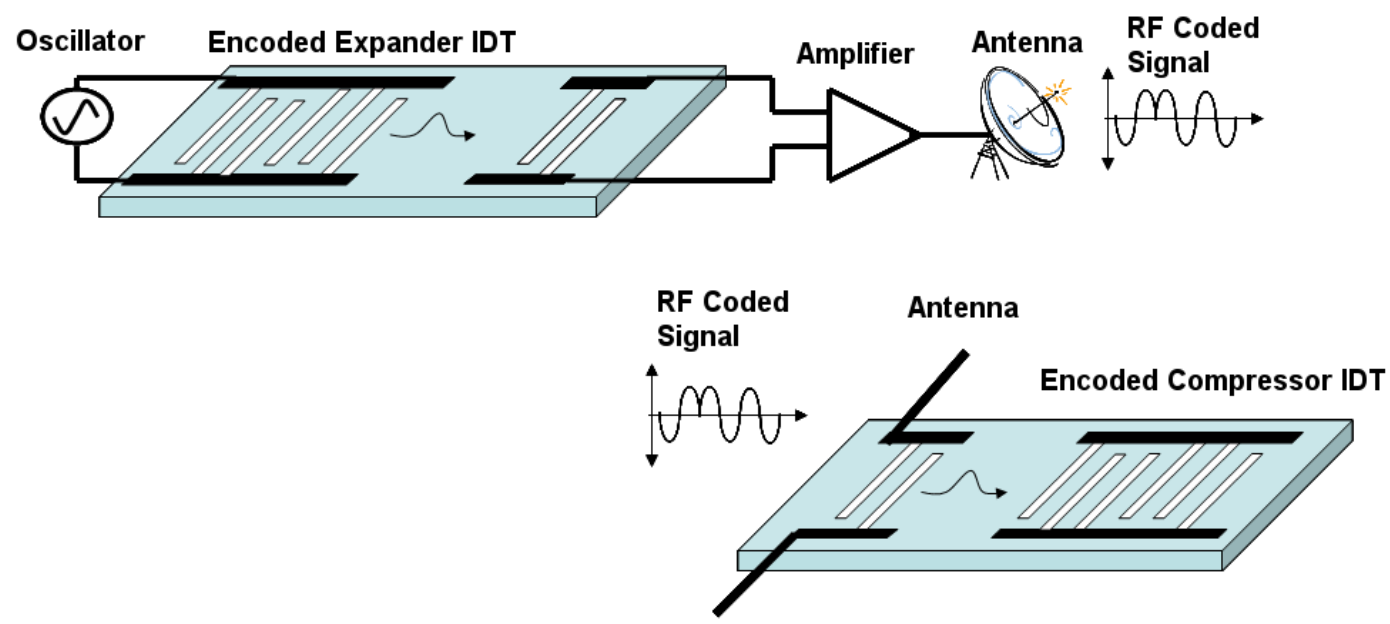

Figure 2. SAW correlator transmitter receiver configuration.

\subsection{Modelling Techniques}

Modelling of SAW correlators is not as straightforward as modelling other commonly used SAW devices due to the large problem domain. It can not be carried out with the same basic software tools that are commercially available for modelling SAW filters and resonators. To aid the correlator design process a few modelling techniques were developed previously. ${ }^{10-12}$ The underlying assumptions, abilities and pitfalls of these techniques, eventually leading to the novel finite element modelling of correlators, will be discussed below.

\subsubsection{Delta Function Modelling}

In the delta function modelling of the correlator, each electrode of the IDT is treated as a delta function. The resultant response of the device is obtained by summing the delta sources. As the correlator is considered as an ideal mathematical element it does not include the harmonic operation of the device, the accurate replication of the output waveforms, and the bulk wave interferences. The model only provides a general understanding of the advantages and disadvantages of different code sequences through the relative insertion loss plots. ${ }^{10,11}$

\subsubsection{Equivalent Circuit Modelling}

In the equivalent circuit modelling approach, the correlator is considered as a three port model, two acoustic ports and one electric port, to describe the response of the device. Transmission lines are employed to replicate 
the behaviour of the acoustic ports. It is a cross field model where the electric field lines are assumed to be normal to the piezoelectric substrate surface.

The model provides the transient response and the peak to side lobe ratio of the correlator. The energy storage effect in electrodes and discontinuities, are important when modelling leaky SAW based devices and the harmonic analysis of the device. However, these effects are not taken into consideration by the model. ${ }^{10-12}$ The frequently used leaky waves or surface transverse waves are much more difficult to describe with simple approximation as Rayleigh waves, since leaky waves are very sensitive to the surface conditions. Though these simulations are computationally intensive, they do not determine the effect of various acoustic modes on the performance of the correlator. Moreover, it is hard to optimise the output response of the device for variable structural dimensions.

\subsubsection{Finite Element Modelling}

The modelling of SAW devices is mathematically equivalent to the resolution of the partial differential equations of piezoelectricity for a given excitation. Finite Element Modelling(FEM) is one of the most accurate method of modelling a SAW device as the complete set of partial differential equations is solved. ${ }^{4}$ This versatile technique automatically takes into account the effects of piezoelectric perturbation, mechanical perturbation, energy storage caused by non-radiating bulk waves, and electric flux leakage from the substrate surface. ${ }^{13}$ Moreover, the strong dependence of correlator performance on the electrode pattern, the second order effects, material type of the substrate and the operating frequency provides a realistic prediction of the complex structures response subject to a RF coded signal excitation.

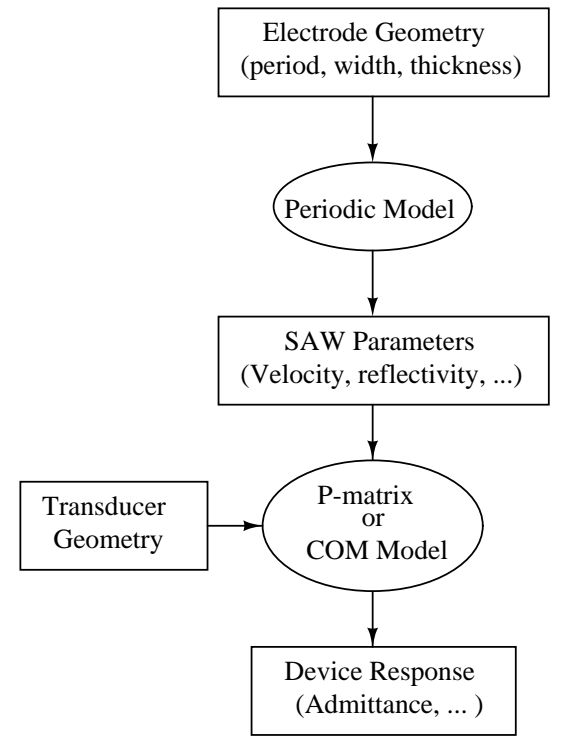

(a)

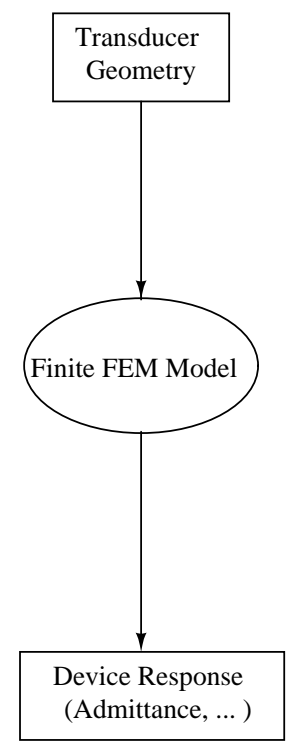

(b)

Figure 3. Two SAW device design approaches for periodic structures (a) and non-periodic sturctures (b).

Two approaches can be used to model SAW devices using FEM. The first approach, shown in Fig. 3.(a), is to consider a structure with infinite periodic gratings, often refered to as periodic structure, and to extract the propagation parameters from the simulation results. These parameter are utilised in scalar models like Coupling of Modes (COM) and P-matrix to model the whole device. The second approach, shown in Fig. 3.(b), is to use a finite model, based on a low level of physical abstraction, and hence the simulation of a SAW device is obtained from the knowledge of its geometrical characteristics. The first approach was employed by the authors in Ref. 8 for modelling SAW devices from the extracted parameters. Because of the non-periodic nature of the SAW correlator the second approach is utilised in this work. 


\section{SAW CORRELATOR DESIGN USING FEM}

The modelling of a 2-dimensional SAW correlator was carried out using ANSYS, a commercially available finite element package. The simulations were aimed to characterise the full scale electromechanical phenomenon of the device, subjected to time and frequency varying excitation. A frequency sweep is performed using harmonic analysis to determine the response of the correlator for different acoustic modes of interest. Apart from the admittance curve and the electric response calculation, analysis of various acoustic modes was carried out with the help of the displacement, electric field and stress contours.

\subsection{Geometry}

A meshed, $5 \times 2$-bit Barker sequence encoded, SAW correlator comprising a piezoelectric substrate and two nonuniform input and output IDT's is shown in the Fig. 4. Both the input and the output transducers have 20 electrodes each. As discussed in Section 2, the input IDT of the structure is modelled as an expander to cause it to generate a coded acoustic wave when a normal pulse or a sinusoidal signal is fed to it. The expander IDT geometry determines the code of the generated acoustic wave. A $5(+++-+) \times 2(++)$ bit Barker sequence is encoded in the output/compressor IDT. This is carried out by coupling the electrodes of the IDT identical to the way they are connected to the positive and negative busbars. The input/expander IDT is an exact replica of the output/compressor IDT when a correlation peak is desired. The response of the device for non-correlating codes can be observed by varying the input IDT finger geometry.

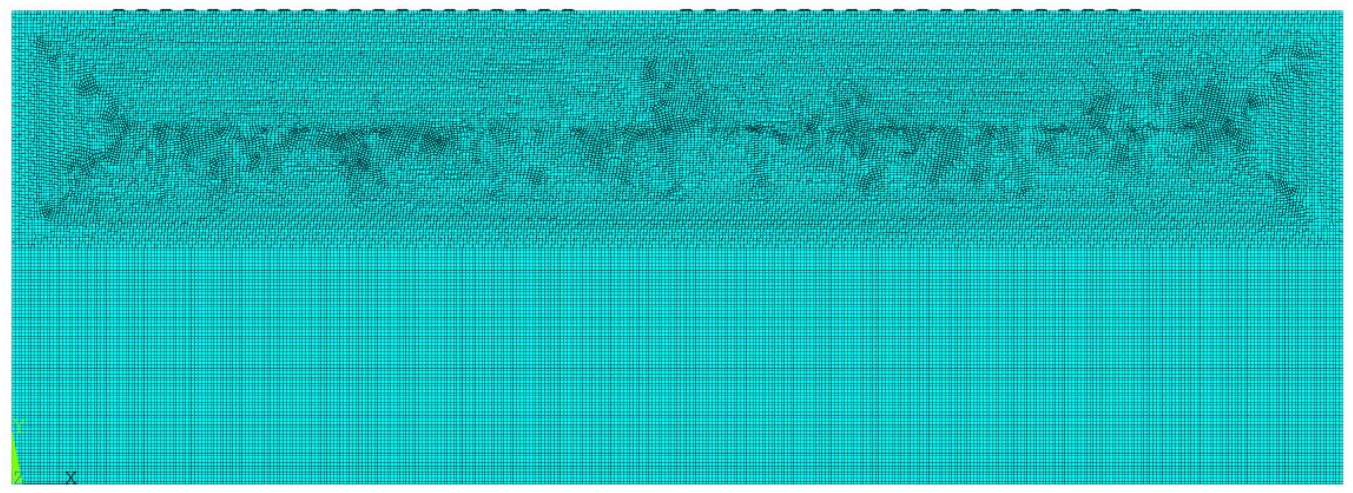

Figure 4. Meshed SAW correlator model. The mesh is discretized to higher densities near the surface than near the bottom as the surface acoustic wave displacements are largest near the substrate surface.

The structure uses a $128^{\circ} \mathrm{YX} \mathrm{LiNbO}_{3}$ piezoelectric substrate of $28 \lambda(\lambda=40 \mu \mathrm{m})$ length, and $10 \lambda$ thickness. Aluminium was used to model the electrodes with a metallization ratio (MR) of 0.4 , and an electrode thickness of $3 \%$. The material properties of piezoelectric substrate and electrodes was obtained from Ref. 14. The separation between the IDT's was chosen to be $2 \lambda$. This was found to be an optimal distance to improve the gain and keep the electromagnetic feedthrough, electromagnetic coupling between the two transducers, under control. ${ }^{5}$

Coupled field element type with 3 degrees of freedom (DOF) was used to model the device. The 3 DOF set provides the voltage, longitudinal and normal displacement information. The reactive charge component of the element was utilised to obtain the admittance curve. The model consists of approximately 212 thousand nodes forming over 70 thousand elements. A denser mesh was generated at the surface and through out the middle of the structure to realise an accurate representation of surface waves. However, the relatively coarser mesh near the bottom surface would still ensure an accurate characterisation of bulk waves as their wavelengths are about twice those of surface waves. ${ }^{3}$

\subsection{Boundary Conditions}

The application of boundary conditions is important to ensure the realistic modelling of the device after the introduction of approximations. Due to large electrode apertures and predominantly lateral propagation of the 
surface waves and bulk waves, a plain strain condition is assumed to reduce the model to a 2D model. ${ }^{15}$ Periodic boundary condition is introduced on the side walls by equating the displacement and electric potential at both ends. Although the periodic side boundary condition was previously used to reduce the simulation domain to one base cell, ${ }^{4,8,15}$ in the proposed model this condition is mainly employed to counter the effect of the reflected waves. The length of the piezoelectric substrate is extended by $2 \lambda$ at the IDT ends to minimise the electromechanical coupling contribution from the the reflected waves. The bottom surface of the correlator is fixed and grounded to reduce the electromagnetic feedthrough. ${ }^{3}$

\section{RESULTS}

The mode propagation properties and the electrical response of the FEM modelled, $5 \times 2$-bit Barker sequence SAW correlator would be described in this section. Modal analysis was performed on the design to identify the freely propagating modes, particularly symmetric and anti-symmetric SAW modes, through the examination of eigenvectors and eigenfrequencies. This was used as a starting point for the harmonic and transient analysis.

\subsection{Harmonic Analysis}

A valuable insight into the excitation properties of the model can be gained from harmonic analysis, where the particular solution of the system of partial differential equations is computed. In this work, the frequency response of correlator is obtained by driving the input IDT electrodes with an alternating voltage of $\pm 1 \mathrm{~V}$ (peak to peak $2 \mathrm{~V})$.

Our interest is to model a low loss correlator, that can power miniature wireless actuators in a secure manner, through the optimisation of design parameters. Therefore, it is not necessary to restrict the results to the modes corresponding to surface acoustic waves. Hence, the harmonic analysis was carried out for a wide frequency range. Depending on the excitation frequency, various acoustic wave modes like the surface acoustic waves or bulk acoustic waves (BAWs) were excited. They can be analysed by observing the contour plots, e.g. displacement, potential, and stress, of the structure.

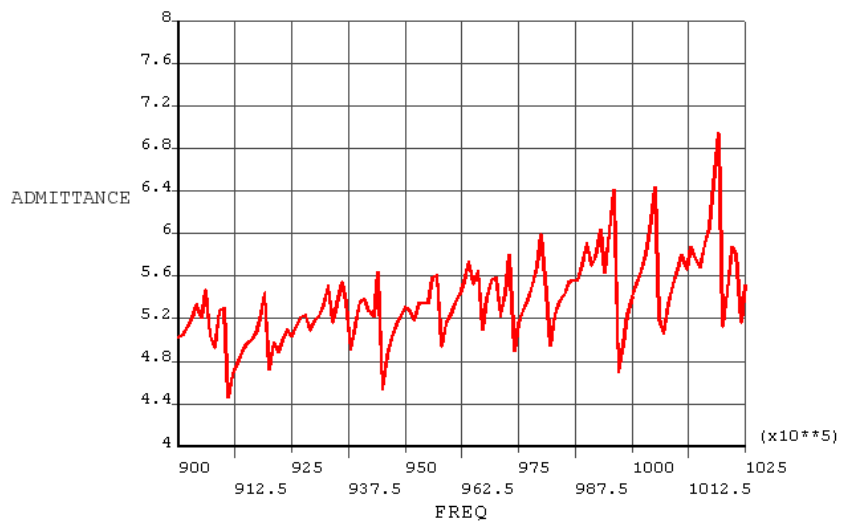

(a)

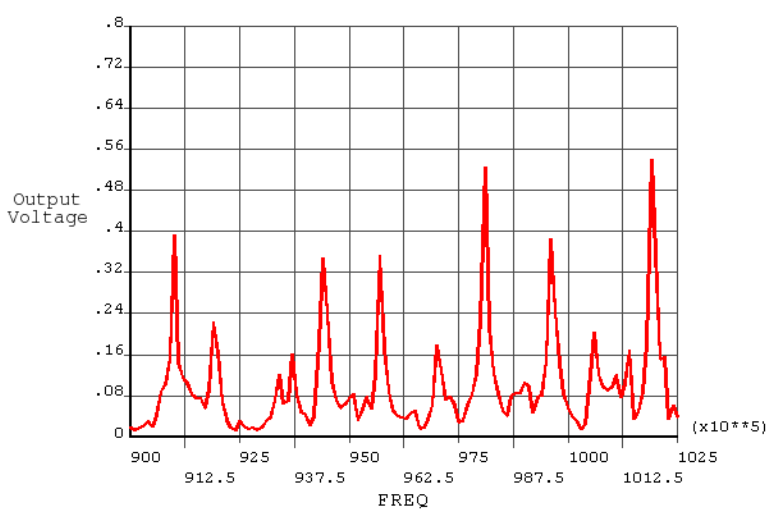

(b)

Figure 5. Frequency response of the correlator for a substrate thickness of $10 \lambda$ (a) Admittance curve. (b) Magnitude of the voltage across the output IDT.

The complex admittance of the device was computed based on the accumulated charge at the electrodes. As can be seen from the Fig. 5.(a), the magnitude of the complex admittance attains a high value at the resonant frequencies and hence exhibits a strong peak. The two admittance peaks to the left of the admittance curve, at frequencies of $90.7 \mathrm{MHz}$ and $91.4 \mathrm{MHz}$, are the one's corresponding to the symmetric and anti-symmetric surface acoustic wave modes. Fig. 6 shows the displacement contour, summation of the $x$ and $y$ displacement components, of the structure for the symmetric SAW mode. In the middle part of the admittance curve, bulk 
acoustic wave modes were observed on the basis of the wave propagation into the bulk of the substrate. An interesting mode, at $100.4 \mathrm{MHz}$, is a leaky surface acoustic wave (LSAW) where both the surface and bulk wave propagations were observed.

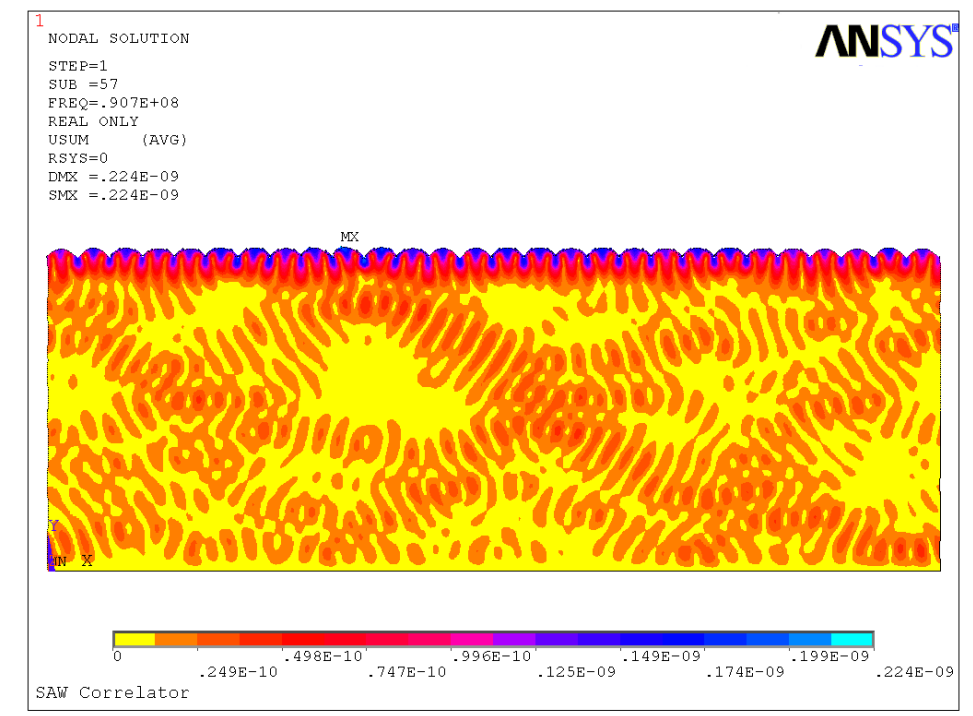

Figure 6. Surface acoustic wave displacement contour for a substrate thickness of $10 \lambda$. The acoustic wave displacement is confined to the top surface of the substrate and, as can be seen from the scale, is in nanometers

Fig. 5.(b) shows the output voltage response of the correlator, when the code matches, for different acoustic modes determined by the excitation frequency. The modes definitely impact the response of the correlator and as can be seen from the Fig. 5.(b), the output response is high for modes other than the SAW modes. Hence, it is desirable to operated the correlator at those bulk and LSAW modes to achieve high electromechanical coupling. Moreover, impact of the variation of the substrate thickness on the correlator response operating at the LSAW mode is described in the following subsection.

\subsection{Effect of the Substrate thickness on the correlator response}

A LSAW propagating along a piezoelectric material with finite thickness is composed of an electric field term and the partial wave components corresponding to the SAW and to the two quasi-shear BAWs. The coupling between these partial waves depends on the properties and orientation of the layer material, the propagation direction as well as on the boundary conditions. The propagation properties of LSAW's on several cuts of $\mathrm{LiNbO}_{3}$ and $\mathrm{LiTaO}_{3}$ substrates were modelled previously with FEM using periodic structures. ${ }^{6,7}$ When fabricating these devices, the thicknesses of the piezoelectric material layer need to be controlled precisely to obtain the correct correlator operating frequency. We will investigate using the proposed modelling approach how the thickness of the piezoelectric substrate is effecting the electromechanical coupling at the LSAW mode.

From the above discussed correlator structure, the piezoelectric substrate thickness was reduced to $5 \lambda$. Then a harmonic analysis was carried out around the LSAW mode excitation frequency of 100.4 MHz. The electromechanical coupling was improved considerably, as is reflected by the increase of the peak value of the voltage across the output IDT, as shown in Fig. 7.(a). The displacement contour for the structure at the LSAW resonant frequency is given in Fig. 7.(b). It can be clearly observed that the particle displacement is not just confined to the top surface as there are bulk wave components propagating through out the thickness of the substrate asserting the propagation characteristics of a LSAW mode.

So far the results are confined to the instance when there is a code match between the input signal, in this model the expander IDT, and the compressor IDT of the correltor. The functionality of the correlator can only 


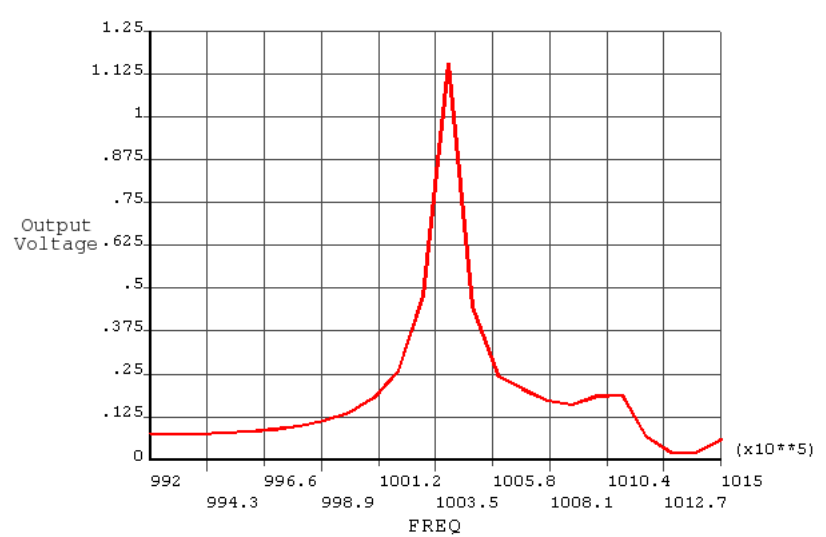

(a)

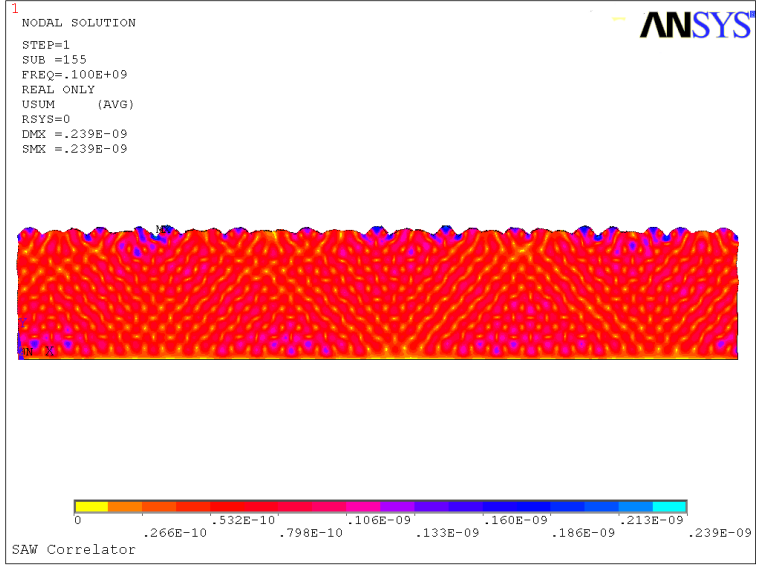

(b)

Figure 7. Frequency response of the correlator for a substrate thickness of $5 \lambda$ (a) Magnitude of the voltage across the output IDT at LSAW mode. (b) LSAW displacement contour.

be verified by observing its response to a mismatched input code. This was carried out by varying the electrode coupling of the expander IDT. The response of the correlator to two different codes, a delay line input and other noncorrelating input, is provided in the Fig. 8, while all other specifications are kept the same.

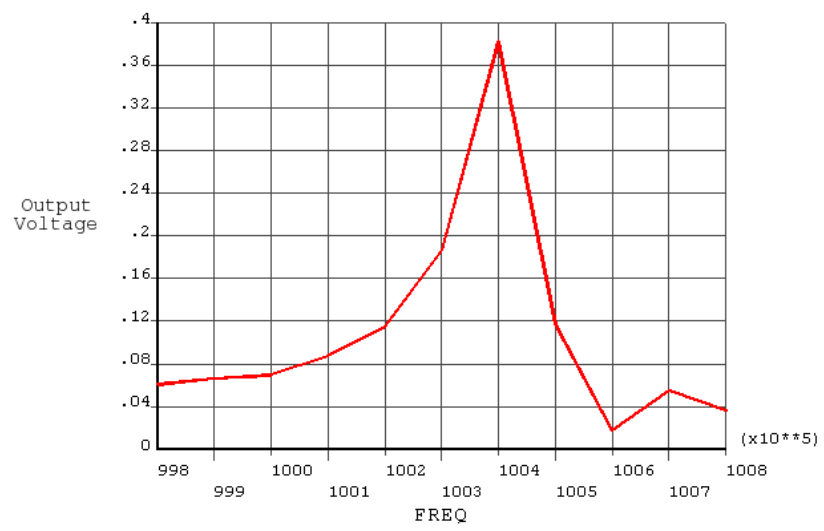

(a)

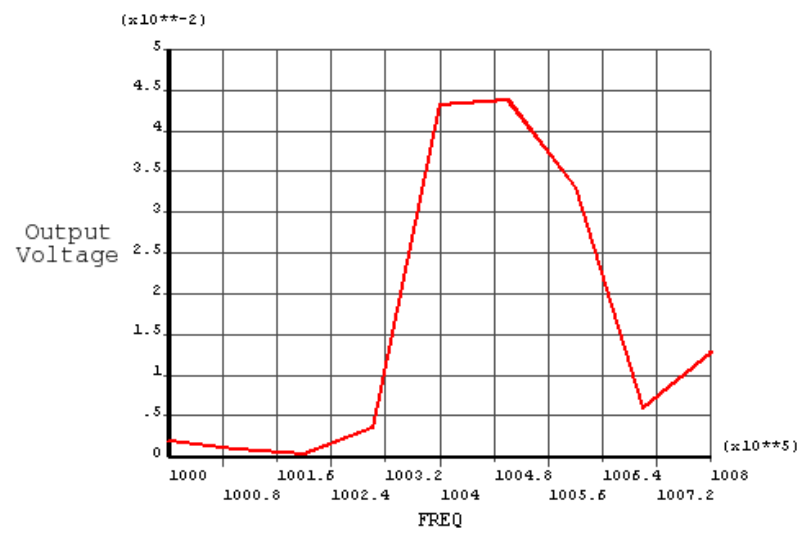

(b)

Figure 8. Voltage across the output IDT when there is a code mismatch (a) delay line input (b) other mismatching input.

By comparing these responses with the correlating peak response of the Fig. 7.(a) it can be established that in addition to the excitation frequency, the correlator's response is determined by the code in the RF signal. Even in the case of a delay line input, when the two codes are very similar, the device response is much less than its response to the matched code, shown in Fig. 7.(a).

\section{CONCLUSION}

A rigorous numerical technique for precise simulation of SAW correlators is presented in this paper. The requirements placed by the current and emerging wireless interrogation applications on these devices such as low insertion loss and high selectivity are taken into consideration. The results discussed in this work include: ( $i$ ) 
Comprehensive FEM modelling of 2-Dimensional, $5 \times 2$-bit Barker sequence encoded SAW correlator, especially the analysis of bulk and LSAW modes (ii) Detailed study of the effect of the LSAW mode on the response of the correlator under an $\mathrm{Al}$ electrode grating in $128^{\circ} \mathrm{YX} \mathrm{LiNbO}_{3}$ piezoelectric substrate, optimization of the substrate thickness to achieve high-Q LSAW correlators, and analysis of difference in electrical response when there is a code mismatch. So finite element analysis provides an effective way of gaining a deeper understanding of the correlator's response to various propagating modes and ultimately in the optimisation of correlator design.

\section{ACKNOWLEDGMENTS}

The authors would like to thank the Australian Research Council (ARC) and the School of Electrical and Electronics Engineering (University of Adelaide) for the funding and support for the project.

\section{REFERENCES}

1. G. Ostermayer, "Correlative signal processing in wireless SAW sensor applications to provide multiple-access capability," in IEEE Trans. on Microwave Theory and Techniques, vol. 49, pp. 809-816, June 2001.

2. R. Brocato, G. Studor, and D. Palmer, "Microsystem packaging of an RF SAW correlator," in Proc. of 55th Electronic Components and Technology Conference, pp. 518-522, May 2005.

3. G. Xu, "Finite element analysis of second order effects on the frequency response of a SAW device," in Proc. of IEEE Ultrasonics Symposium, pp. 187-190, October 2000.

4. M. Hofer, N. Finger, G. Kovacs, J. Schölmer, S. Zaglmayr, U. Langer, and R. Lerch, "Finite-Element simulation of wave propagation in periodic piezoelectric SAW structures," in IEEE Trans. on Ultrasonics, Ferroelectrics, and Frequency Control, vol. 53, June 2006.

5. S. Ippolito, K. Kalantar-Zadeh, D. Powell, and W. Wlodarski, "A 3-dimensional finite element approach for simulating acoustic wave propagation in layered SAW devices," in Proc. of IEEE Ultrasonics Symposium, pp. 303-306, February 2003.

6. M. Hofer, N. Finger, G. Kovacs, J. Schölmer, U. Langer, and R. Lerch, "Finite element simulation of bulk and Surface Acoustic Wave (SAW) Interaction in SAW Devices," in Proc. of IEEE Ultrasonics Symposium, pp. 53-56, 2002.

7. T. Makkonen, V. Plessky, W. Steichen, V. Grigorievski, M. Solal, and M. Salomaa, "Longitudinal leaky

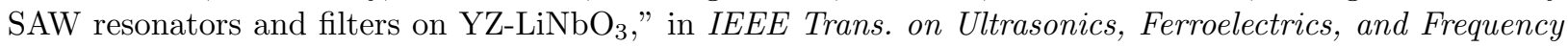
Control, vol. 53, pp. 393-401, February 2006.

8. A. Tikka, S. Al-Sarawi, and D. Abbott, "SAW parameter extraction using Finite Element Analysis," in 2nd International Conference on Sensing Technology, November 2007. Accepted.

9. A. Tikka, S. Al-Sarawi, D. Abbott, M. Wong, and J. Schutz, "Improving the security and actuation of wireless controlled microvalve," in Proc. of SPIE Smart Structures, Devices, and Systems conference, vol. 6414, January 2007.

10. R. Brocato, Programmable SAW Development, Sandia National Laboratories, http://www.sandia.gov/mstc/products/usystemsprod/rfandoptosystems/saw.html, October 2004. Last accessed: Nov, 2007.

11. C. K. Campbell, Surface Acoustic Wave Devices for Mobile and Wireless Communications, ch. 4. Academic Press: Boston, 1998.

12. K. Hashimoto, Surface Acoustic Wave Devices in Telecommunications Modelling and Simulation, ch. 3. Springer, 2000.

13. K. Hasegawa, K. Inagawa, and M. Koshiba, "Extraction of all coefficients of coupled-mode equations for natural, single phase by hybrid finite element method," in IEEE Trans. on Ultrasonics, Ferroelectrics, and Frequency Control, vol. 48, September 2001.

14. B. Auld, Acoustic Fields and Waves in Solids, ch. Appendix 2. Rober E. Krieger, 1990 (2 ${ }^{\text {nd }}$ Edition).

15. Y. Yong, "Analysis of periodic structures for BAW and SAW resonators," in Proc. of IEEE Ultrasonics Symposium, pp. 781-790, 2001. 